\title{
Finite Difference Approximations for Fractional Reaction-Diffusion Equations and the Application In PM2.5
}

\author{
Changping $\mathrm{Xie}^{1, \mathrm{a}}$, Lang $\mathrm{Li}^{1, \mathrm{~b}}$, Zhongzhan Huang ${ }^{1, \mathrm{c}}$, Jinyan $\mathrm{Li}^{1, \mathrm{~d}}$, PengLiang $\mathrm{Li}^{1, \mathrm{e}}$ \\ Shaomei Fang ${ }^{1, \mathrm{f},}$ \\ ${ }^{1}$ College of Mathematics and Informatics, South China Agricultural University, Guangzhou 510642, \\ China \\ a229430694@qq.com, ${ }^{b}$ lilang05422@163.com, ${ }^{c}$ 2644696581@qq.com, duduwoxing@126.com, \\ e3290236818@qq.com, 'dz90@scau.edu.cn \\ ${ }^{*}$ Corresponding author
}

Keywords: PM2.5; fractional reaction-diffusion equations; Crank-Nicolson method; numerical simulation.

Abstract. In this paper, fractional reaction-diffusion equations are used to model the diffusion of PM2.5 in the air. First, based on the shifted Grünwald formula, we propose the fractional Crank-Nicolson method to solve the fractional reaction-diffusion equations. Then we prove the existence and uniqueness of numerical solutions, and establish the stability and convergence of the method. Furthermore, numerical examples are also provided to show the efficiency of the method. Finally, the diffusion of PM2.5 in Guangzhou is simulated by using this method under appropriate parameters .

\section{Introduction}

With the rapid development of economy and constant improvement of human's requirements of living environment, the air pollution, especially PM2.5, has drawn much attention in China. The so called PM2.5, particles of aerodynamic diameter less than 2.5 micrometers, can lead to hazy weather and cause health problems [1]. Therefore, it is important and necessary to predict precisely the PM2.5 concentrations for the control of air pollution and the improvement of the human living conditions.. However, it is not adequate to model and predict the PM2.5 concentrations using traditional methods when the fire or explosion takes place in somewhere. For this case, it is crucial to find a suitable method or model to predict the PM2.5 concentrations.

Fractional reaction-diffusion equations can be regarded as the generalization of classical reaction-diffusion equations [2] and have been successfully applied to model problems in physics [3], biology [4], finance [5]. In this paper, we will use the following fractional reaction-diffusion equation to describe the diffusion of PM2.5:

$$
\frac{\partial u(x, t)}{\partial t}=-b(x) \frac{\partial u(x, t)}{\partial x}+a(x) \frac{\partial^{\alpha} u(x, t)}{\partial x^{\alpha}}-c(x) u(x, t)+f(x, t), \quad L<x<R, t>0,
$$

subject to the initial and Dirichlet boundary conditions given by

$$
\begin{aligned}
& u(x, 0)=q(x), \\
& L \leq x \leq R, \\
& u(t, L)=r(t), u(t, R)=s(t), \\
& t>0 \text {. }
\end{aligned}
$$

where the diffusion coefficient $a(x) \geq 0$, wind speed $b(x) \geq 0$, and decay coefficient $c(x) \geq 0$ are continuous, $f(x, t)$ is a continuous function on $[L, R] \times[0, T]$ which represents sources and sinks. $u(x, t)$ is the pollutant concentration.

Here $\frac{\partial^{\alpha} u(x, t)}{\partial x^{\alpha}}(1<\alpha<2)$ is a Riemann fractional derivative of order $\alpha$, which is defined by[6]

$$
\frac{\partial^{\alpha} u(x, t)}{\partial x^{\alpha}}=\frac{1}{\Gamma(2-\alpha)} \frac{\partial^{2}}{\partial x^{2}} \int_{L}^{x} \frac{u(\xi, t)}{(x-\xi)^{\alpha-1}} d \xi .
$$


For this equation, many researchers have studied the existence and uniqueness of the solution and acquired good results [6-7]. While to establish the numerical solution for this equation is also attractive. There are two ways to discretize the fractional derivatives. One is shifted Grünwald formula [8], the other is finite difference scheme [9]. This paper takes the first way to approximate the fractional space derivatives, and construct the Crank-Nicolson scheme. Below we prove the existence of the numerical solution and then we analyze the stability and convergence of the scheme. An example with known exact solution is also presented to test the efficiency of the scheme. Finally, we simulate the diffusion of PM2.5 in Guangzhou by choosing appropriate parameters.

\section{The Crank-Nicolson scheme for the fractional reaction-diffusion equation}

In this section, we propose the Crank-Nicolson numerical approximation scheme based on the shifted Grünwald formula [10-11], which is defined as

$$
\frac{\partial^{\alpha} u(x, t)}{\partial x^{\alpha}}=\frac{1}{l^{\alpha}} \lim _{M \rightarrow \infty} \sum_{k=0}^{M} g_{k} u(x-(k-1) l, t),
$$

where $M$ is a positive integer, $l=\frac{x-L}{M}$ and $g_{k}=\frac{\Gamma(k-\alpha)}{\Gamma(-\alpha) \Gamma(k+1)}, k=1,2,3, \cdots$.

We define $h$ to be the grid size in spatial direction with $K=\frac{R-L}{h}, x_{i}=L+i h, i=0,1,2, \cdots K$; and $\tau$ to be the grid size in time direction, with $N=\left[\frac{T}{\tau}\right], t_{n}=\tau n, n=0,1,2 \cdots, N$.

Denote $a_{i}=a\left(x_{i}\right), b_{i}=b\left(x_{i}\right), c_{i}=c\left(x_{i}\right), f_{i}^{n+1 / 2}=f\left(x_{i}, t_{n+1 / 2}\right), D_{x} u_{i}^{n}=\left(u_{i}^{n}-u_{i-1}^{n}\right) / h$, $\theta_{x} u_{i}^{n}=\frac{1}{h^{\alpha}} \sum_{k=0}^{i+1} g_{k} u_{i-k+1}^{n}, u_{i}^{n}$ is the numerical solution of Eqs. (1)-(3), we have the following scheme:

$$
\begin{array}{ll}
\frac{1}{\tau}\left(u_{i}^{n+1}-u_{i}^{n}\right)=-\frac{b_{i}}{2}\left(D_{x} u_{i}^{n+1}+D_{x} u_{i}^{n}\right)+\frac{a_{i}}{2}\left(\theta_{x} u_{i}^{n+1}+\theta_{x} u_{i}^{n}\right)-\frac{c_{i}}{2}\left(u_{i}^{n+1}+u_{i}^{n}\right)+f_{i}^{n+1 / 2}, \\
& 1 \leq i \leq K-1,1 \leq n \leq N \\
u_{i}^{0}=q\left(x_{i}\right), & 0 \leq i \leq K \\
u_{0}^{n}=r\left(t_{n}\right), u_{K}^{n}=s\left(t_{n}\right), & 1 \leq n \leq N
\end{array}
$$

Therefore, Eqs. (5)-(7) can be written as the following matrix form:

$$
(I+A) \underline{U}^{n+1}=(I-A) \underline{U}^{n}+\tau \underline{F}^{n+1 / 2}
$$

where

$$
\underline{C}^{n}=\left[\begin{array}{c}
c_{1}^{n} \\
c_{2}^{n} \\
\vdots \\
c_{K-1}^{n}
\end{array}\right], \tau \underline{F}^{n+\frac{1}{2}}=\left[\begin{array}{c}
\tau f_{1}^{n+1 / 2}+\left(E_{1}+B_{1} g_{2}\right)\left(r\left(t_{n+1}\right)+r\left(t_{n}\right)\right) \\
\tau f_{2}^{n+1 / 2}+B_{2} g_{3}\left(r\left(t_{n+1}\right)+r\left(t_{n}\right)\right) \\
\vdots \\
\tau f_{K-2}^{n+1 / 2}+B_{K-2} g_{K-1}\left(r\left(t_{n+1}\right)+r\left(t_{n}\right)\right) \\
\tau f_{K-1}^{n+1 / 2}+B_{K-1} g_{K}\left(r\left(t_{n+1}\right)+r\left(t_{n}\right)\right)+B_{K-1} g_{0}\left(s\left(t_{n+1}\right)+s\left(t_{n}\right)\right)
\end{array}\right],
$$

and matrix $A=\left(A_{i, j}\right)_{(K-1) \times(K-1)}$ is defined as follows:

$$
A_{i, j}=\left\{\begin{array}{cc}
0 & j \geq i+2 \\
-g_{0} B_{i} & j=i+1 \\
E_{i}-g_{1} B_{i}+C_{i} & j=i \\
-E_{i}-g_{2} B_{i} & j=i-1 \\
-g_{i-j+1} B_{i} & j \leq i-2
\end{array}\right.
$$


with $B_{i}=\frac{a_{i} \tau}{2 h^{\alpha}}, E_{i}=\frac{b_{i} \tau}{2 h}, C_{i}=\frac{C_{i} \tau}{2}$.

\section{Stability and convergence}

Theorem 1. The Crank-Nicolson scheme defined by Eqs. (5)-(7) has a unique solution and is unconditional stable for all $1<\alpha<2$.

Proof. Let $\lambda$ be an eigenvalue of the matrix $\mathrm{A}$, and $A X=\lambda X$ for some nonzero vector $X$. Choose $i$ such that $\left|x_{i}\right|=\max \left\{\left|x_{j}\right|: j=0,1,2, \cdots K-1\right\}$. Then $\sum_{j=0}^{K-1} A_{i, j} x_{j}=\lambda x_{i}$, and therefore

$$
\lambda=A_{i, i}+\sum_{j=0, j \neq i}^{K-1} A_{i, j} \frac{x_{j}}{x_{i}} .
$$

Substituting the values of $A_{i, j}$ into (10) we have

$$
\lambda=E_{i}-g_{1} B_{i}+C_{i}-g_{0} B_{i} \frac{x_{i+1}}{x_{i}}-\left(E_{i}+g_{2} B\right) \frac{x_{i-1}}{x_{i}}-\sum_{j=1}^{i-2} g_{i-j+1} B_{i} \frac{x_{j}}{x_{i}}=E_{i}\left(1-\frac{x_{i-1}}{x_{i}}\right)-B_{i}\left(g_{1}+\sum_{j=1, j \neq i}^{i+1} g_{i-j+1} \frac{x_{j}}{x_{i}}\right)+C_{i} \text {. }
$$

Then by [11], we can see that $\sum_{k=0, k \neq 1}^{j} g_{k} \leq-g_{1}(j=0,1,2, \cdots)$. Therefore

$$
\sum_{j=1, j \neq i}^{i+1} g_{i-j+1} \frac{x_{j}}{x_{i}} \leq \sum_{j=1, j \neq i}^{i+1} g_{i-j+1}\left|\frac{x_{j}}{x_{i}}\right| \leq \sum_{j=1, j \neq i}^{i+1} g_{i-j+1} \leq-g_{1} .
$$

Since $E_{i}, B_{i}, C_{i}$ are non-negative, it follows that

$$
E_{i}\left(1-\frac{x_{i-1}}{x_{i}}\right)-B_{i}\left(g_{1}+\sum_{j=1, j \neq i}^{i+1} g_{i-j+1} \frac{x_{j}}{x_{i}}\right)+C_{i} \geq 0
$$

Then we have $\lambda \geq 0,1+\lambda \geq 1$, which means that every eigenvalue of $I+A$ is no less than 1 . Therefore, the spectral radius of $(I+A)^{-1}$ satisfies $\rho\left((I+A)^{-1}\right) \leq 1$. Thus we prove that the Eqs. (5)-(7) has a unique solution.

To prove the unconditional stability of the scheme, we assume $\varepsilon^{0}$ to be the error in $\underline{U}^{0}$, then it will result in an error in $\underline{U}^{n}$ given by $\varepsilon^{n}=\left[(I+A)^{-1}(I-A)\right]^{n} \varepsilon^{0}$. We denote by $\eta$ an eigenvalue of $(I+A)^{-1}(I-A)$, then we have $|\eta| \leq 1$. Thus $\left\|\varepsilon^{n}\right\|_{\infty} \leq\left\|\varepsilon^{0}\right\|_{\infty}$. So the method is unconditional stable.

Theorem 2. The scheme defined by Eqs. (5)-(7) with $1<\alpha<2$ is unconditional convergent.

Proof. In light of [12], we can see the local truncation error of Eqs. (5)-(7) is $O\left(h+\tau^{2}\right)$, therefore the scheme is consistent. Then according to Theorem 1 and Lax equivalence theorem [12], we can show the method is convergent and the order of convergence is $O\left(h+\tau^{2}\right)$.

\section{A numerical example}

The following fractional reaction-diffusion equation

$$
\frac{\partial u(x, t)}{\partial t}=-b(x) \frac{\partial u(x, t)}{\partial x}+a(x) \frac{\partial^{1.5} u(x, t)}{\partial x^{1.5}}-c(x) u(x, t)+f(x, t), 0<x<1, t>0,
$$

was considered with the initial and boundary condition

$$
u(x, 0)=x^{2}, 0 \leq x \leq 1 ; u(0, t)=0, u(1, t)=e^{-t}, t>0 .
$$

Here we take

$$
b(x)=\frac{5}{4} x^{2}, a(x)=\frac{1}{4} \Gamma\left(\frac{1}{2}\right) x^{3 / 2}, c(x)=\frac{x}{2}, f(x, t)=\left(3 x+\frac{1}{2}\right) x^{2} e^{-t} .
$$


Then the exact solution to this fractional equation is given by $u(x, t)=x^{2} e^{-t}$.

Fig. 1 shows the numerical solution at time $t=1$ obtained from the Crank-Nicolson method Eq. (8) discussed above with $h=1 / 40, \tau=1 / 20$. As can be seen from Fig. 1, this numerical solution compares well with the exact analytic solution.

Table 1 shows that as the number of spatial steps is quadrupled and time steps is doubled, the maximum error is quadrupled, as expected from the order $O\left(h+\tau^{2}\right)$ of the convergence of the scheme.

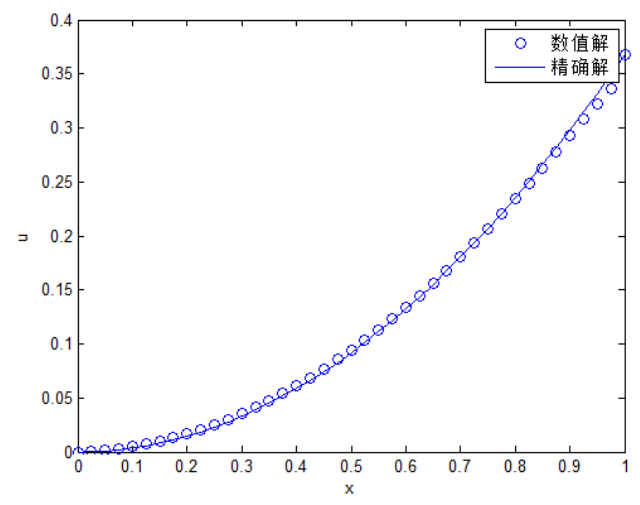

Fig. 1 Comparing the numerical solution with the exact solution ( $h=1 / 40, \tau=1 / 20$ )

Table 1 Maximum error behavior for the example problem

\begin{tabular}{llll}
\hline$t$ & $(\tau, h)=(1 / 20,1 / 8)$ & $(\tau, h)=(1 / 40,1 / 32)$ & Error rate \\
\hline 0.5 & $2.6507 e-2$ & $7.3562 e-3$ & 3.61 \\
1.0 & $2.6766 e-2$ & $6.2247 e-3$ & 4.30 \\
1.5 & $2.1095 e-2$ & $5.2372 e-3$ & 4.03 \\
2.0 & $1.5121 e-2$ & $3.9448 e-3$ & 3.83 \\
\hline
\end{tabular}

\section{Numerical simulation for the diffusion of PM2.5}

Below we will give a numerical simulation for the diffusion of PM2.5 in Guangzhou by choosing proper parameters.

Here we choose the diffusion parameter of $\mathrm{SO}_{2}$ to replace the diffusion parameter of PM2.5 i.e. $a(x)=1.22 \times 10^{-5} \mathrm{~m}^{2} / \mathrm{s}$, as the diffusion parameter of PM2.5 is hard to determine and $\mathrm{SO}_{2}$ is one of the most important chemical compositions of PM2.5 in Guangzhou[13-14]. For the wind velocity, from China meteorological data sharing service system, we know the average speed of wind in Guangzhou, with scale $0-3$, is less than $5.4 \mathrm{~m} / \mathrm{s}$. Without loss of generality, we take the direction of wind as the $x$-axis, and the speed of wind is $5 \mathrm{~m} / \mathrm{s}$, i.e. $b(x)=5 \mathrm{~m} / \mathrm{s}$. Finally, we determine decay rate of PM2.5. It can be approximate to 0 due to the fact that PM2.5 is difficult to disappear automatically without control. i.e. $c(x)=0$.

In this paper, we take the fractional order equal to 1.5. i.e. $\alpha=1.5$. Then we have

$$
\frac{\partial u}{\partial t}=-5 \frac{\partial u}{\partial x}+1.22 \times 10^{-5} \frac{\partial^{1.5} u}{\partial^{1.5} x}+f(x, t), 0<x<1, t>0,
$$

with initial boundary conditions

$$
u(x, 0)=e^{-x}, 0 \leq x \leq 1, ; u(0, t)=e^{\frac{1}{2} t}, u(1, t)=e^{-1+\frac{1}{2} t}, t>0,
$$

and the source/sink function

$$
f(x, t)=-\frac{9}{2} e^{-x+\frac{1}{2} t}
$$

Here we choose $h=0.1, \tau=0.1$. Fig. 2 and Fig. 3 present the solution surface and the contour lines of PM2.5 concentration respectively. As can be seen clearly from Fig. 2, PM2.5 concentrations decrease with the increase in $x$ and increase with the increase in $t$. This indicates that the speed of pollutants released is more than the speed of diffusion. On the other hand, from Fig. 3, we can see the 
contour is getting more and more sparse with the increase of $x$ for fixed $t$, which indicates the higher the concentrations of PM2.5, the faster of the concentrations decreased and the smaller range with high PM2.5 concentrations. Moreover, Fig. 3 also shows the contour is getting more and more intensive with the increase in $t$ for fixed $x$, which means PM2.5 concentrations increase more rapidly with the increase in $t$.

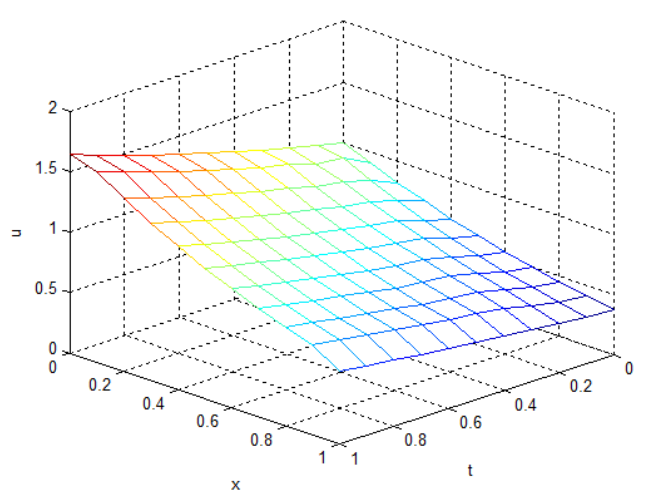

Fig. 2. The distribution of PM2.5

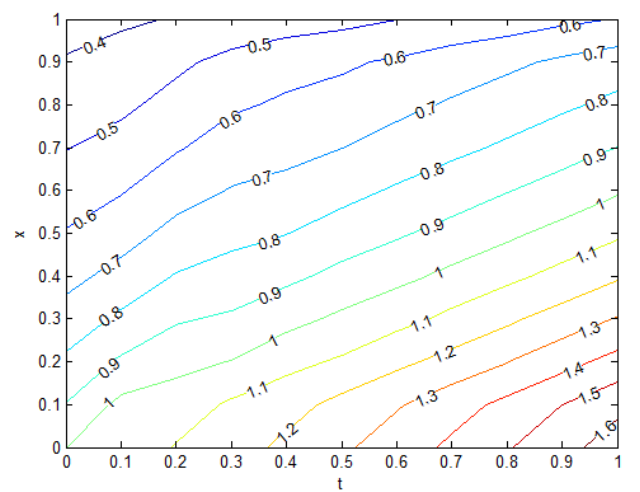

Fig. 3. The contour lines of PM2.5 concentrations

\section{Acknowledgements}

This work was financially supported by the Guangdong special funds for cultivation of technological innovation by university students (pdjh2015b0094) and the NSFC (11271141).

\section{References}

[1] N. Martinelli, O. Olivieri and D. Girelli: Eur. J. Intern. Med., 295-302 (2013), p. 24.

[2] R. Metzler and J. Klafter: Phys. Rep. 1-77 (2000), p. 339 (1).

[3] Y. Rossilihin and M. Shitikova: Applied Mechanics Reviews. 15-67 (1997) p. 50.

[4] S.B. Yuste and K. Lindenberg: Phys. Rev. Lett.118-301 (2001), p. 87 (11).

[5] E. Scalas, R. Gorenflo and F. Mainardi: Statistical Mechanics and its Applications.376-384(2000), p. 284(1).

[6] I. Podlubny: Fractional Differential Equations (Academic Press, New York 1999).

[7] D.F. Mainar and Y. Luchko: Fractional Calculus and Applied Analysis.153-192 (2001), p. 4(2).

[8] Jinghua Chen and Fawang Liu: Journal of Xiamen University (Natural Science) (In Chinese).466-469 (2006), p. 45 (4).

[9] M.M. Meerschaert, H.P. Scheffler and C. Tadjeran: J Comput Phys.249-261(2006), p. 21(1).

[10]M. M. Meerschaert and C. Tadjeran: Journal of Computational and Applied Mathematics.65-77(2003), p. 172(1).

[11] C. Tadjeran, M.M. Meerschaert and H.P. Scheffler: J Comput Phys. 205-213(2006), p. 213(1).

[12] R.D Richtmyer, K.M Morton: Difference Methods for Initial Value Problems.2nd ed. (John Wiley \& Sons, New York 1980).

[13] C.Y. Chen, et al: Environmental Monitoring in China. 61-64(2006), p. 05.

[14]Z.L Cheng, K.S Lam, L.Y Chan, T. Wanga nd K.K Cheng: Atmospheric Environment. 2771-2783(2000), p. 3417. 